مجلة جامعة سبها للعلوم البحتة والتطبيقية

Sebha University Journal of Pure \& Applied Sciences

Journal homepage: www.sebhau.edu.ly/journal/index.php/jopas

\title{
On A Matrix Hypergeometric Differential Equation
}

Salah Hamd ${ }^{1}$, Faisal Saleh Abdalla ${ }^{2}$, Ahmed Shletiet ${ }^{3}$

${ }^{1}$ Department of Mathematics, College of Education, Omar Al-Mukhtar University, Al-Bayda,Libya

${ }^{2}$ Department of general science, College of mechanical engineering technology - Benghazi, Benghazi,Libya

${ }^{3}$ Department of Mathematics, faculty of science, University of Ajdabiya, Ajdabiya, Libya.

\section{Keywords:}

Special functions

A matrix Hypergeometric

differential Equation.

Matrix.

Gamma function.

Jordan Canonical Form.

\begin{abstract}
A B S T R A C T
In this paper we consider a matrix Hypergeometric differential equation, which are special matrix functions and solution of a specific second order linear differential equation. The aim of this work is to extend a well known theorem on Hypergeometric function in the complex plane to a matrix version, and we show that the asymptotic expansions of Hypergeometric function in the complex plane" that are given in the literature are special members of our main result. Background and motivation are discussed.
\end{abstract}

$$
\begin{aligned}
& \text { حل المعادلة التفاضلية لدالة فوق هندسية في الصيورة المصفوفية } \\
& \text { صلاح حمد و فيصل صالح عبد الله و وأحمد شليتيت3 } \\
& \text { 1قسم الرياضيات، كلية التربية، جامعة عمر المختار، ليبيا }
\end{aligned}
$$

\begin{tabular}{|c|c|}
\hline 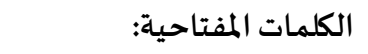 & 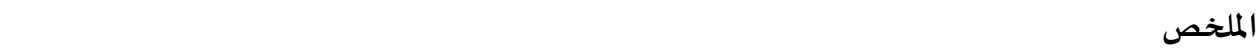 \\
\hline 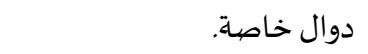 & في هذا البحث تمت دراسة المعادلة التفاضلية لدالة فوق هندسية في الصورة المصفوفية والتي تمثل دالة \\
\hline 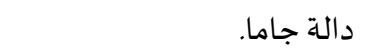 & مصفوفية خاصة لحل معادلة تفاضلية خطية من الرتبة الثانية. والهدف من هذه الدراسة هو تعميم نظرية \\
\hline صيغة جوردان القانونية. & مشهورة على الدوال فوق الهندسية لتكون في الصيغة المصفوفية بدلا من الصيورة التي كانت عليها في المستوى \\
\hline مصفوفة. & المركب، بالأضافة إلى ذلك تم أثبات تقريب المقرب للدوال فوق الهندسية في المستوى المركب والتي تم عرضهها في \\
\hline معادلة تفاضلية فوق هندسية. & بحوث سـابقة كحالة خاصة من النتيجة التي توصلنا إليها. خلال هذا البحث تم مناقشة الاساسيات والدوافع \\
\hline
\end{tabular}

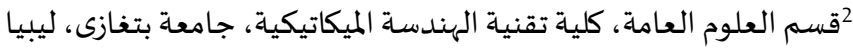

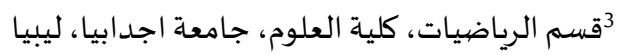

\section{Introduction}

Generalization and extension of scalar special functions to matrix special functions have been developed in the past two decades The Gamma matrix function, whose eigenvalues are all in the right open half-plane is presented and investigated by L. J'odar, J. Cort'es [1] for matrices in $C^{r x r}$. Hermite matrix polynomials are introduced and discussed by L. J'odar et al [2] and some of their properties are provided in E. Defez, L. J'odar [3]. Other classical orthogonal polynomials as Laguerre and Chebyshev have been extended to orthogonal matrix polynomials, and some results have been studied in L. J'odar, J. Sastre [4] and E. Defez, L. J'odar [5]. Relations between the Beta, Gamma and the Hypergeometric matrix function are given in L. J'odar, J. G. Cort'es [6] and R. S. Batahan [7]. These special functions of matrices have developed an important tool in both theory and applications. The main our goal is that, some cases of the asymptotic expansions of $2 \mathrm{~F} 1(\mathrm{a}, \mathrm{b} ; \mathrm{c} ; \mathrm{z})$ have been provided in the literature, they are all limited by a narrow domain of validity in the complex plane of the variable. Overcoming this restriction, we provide new asymptotic expansion for the matrix hypergeometric function .The order of presentation in this article is as follows. In section 2 we provide basic necessary notation, definitions and auxiliary theorems that need to be cited in the sequel. In section 3 we provide our main results.

\section{Preliminaries}

In this part we elaborate on some necessary language that is adopted from L. J'odar, J. Sastre [4] and N. J. Higham [8]. Denote by

*Corresponding author:

E-mail addresses: salahh92@gmail.com, (F. S. Abdalla2) faisal@ceb.edu.ly, (A. Shletiet) Ahmed.shletiet@uoa.edu.ly Article History : Received 27 March 2021-Received in revised form 25 May 2021 - Accepted 01 june 2021 
$\lambda_{1}, \lambda_{2} \cdots, \lambda_{\mathrm{n}}$ the distinct eigenvalues of a matrix $\mathrm{P} \in \mathrm{C}^{\mathrm{r} \times \mathrm{r}}$. The spectrum $\sigma(\mathrm{P})$ of $\mathrm{P} \in \mathrm{C}^{\mathrm{r} \times \mathrm{r}}$, denotes the set of all the eigenvalues of $\mathrm{P}$. We put $\gamma(\mathrm{P})$. and $\varrho(\mathrm{P})$ the real numbers

$$
\gamma(P)=\max \{\operatorname{Re}(\lambda): \lambda \in \sigma(P)\}
$$$$
\varrho(P)=\min \{\operatorname{Re}(\lambda): \lambda \in \sigma(P)\}
$$

holomorphic function $\mathrm{f}(\lambda)$ at a point was defined as a regular analytic function in a neighborhood of the point, see e.g. W. Wasow [9]. It is called holomorphic in a set if it is holomorphic at every point of the set. A matrix is called holomorphic if every entry of it is a holomorphic function. If $\boldsymbol{f}(\boldsymbol{\lambda})$ and $\boldsymbol{g}(\lambda)$ are homomorphic function of the complex variable $\lambda$, which are defined in an open set $\Omega$ of the complex plane, and $\mathrm{P}$ is matrix in $\mathrm{C}^{\mathrm{r} \times \mathrm{r}}$ with $\sigma(\mathrm{P}) \subset \Omega$, than from the properties of the matrix functional calculus, see N. Dunford, J. Schwartz [10], it follows that

$$
\boldsymbol{f}(\boldsymbol{P}) \boldsymbol{g}(\boldsymbol{P})=\boldsymbol{g}(\boldsymbol{P}) \boldsymbol{f}(\boldsymbol{P})
$$

A set of complex numbers is called positive stable if all the elements of the set have positive real part and a square matrix $\mathrm{P}$ is called positive stable if $\sigma(\mathrm{P})$ is positive stable.

If $\mathrm{P}$ is a positive stable matrix in $\mathrm{C}^{\mathrm{r} \times \mathrm{r}}$, than $\Gamma(\mathrm{P})$ is well defined, see L. J'odar, J. G. Cort'es [1]

$$
\Gamma(P)=\int_{0}^{\infty} e^{-t} t^{p-1} d t
$$

If $\boldsymbol{f}(\boldsymbol{P})$ is well defined and $\mathrm{T}$ is an invertible matrix in $\mathrm{C}^{\mathrm{r} \times \mathrm{r}}$, then

$$
f \llbracket\left(\mathbf{T P T} \boldsymbol{T}^{-1}\right) \rrbracket=\boldsymbol{T} \boldsymbol{f}(\boldsymbol{P}) \boldsymbol{T}^{-1}
$$

It is a standard result that for any matrix $P \in C^{r \times r}$ there exist a nonsingular matrix $\mathrm{T} \in \mathrm{C}^{\mathrm{r} \times \mathrm{r}}$ such that

$T^{-1} P T=J=\operatorname{diag}\left(J_{1}, J_{2}, \ldots \ldots, J_{N}\right)$

Where

$$
\mathrm{J}_{\mathrm{k}}=\mathrm{J}_{\mathrm{k}}\left(\lambda_{\mathrm{k}}\right)=\left[\begin{array}{ccccc}
\lambda_{\boldsymbol{k}} & \mathbf{1} & \mathbf{0} & \ldots & \mathbf{0} \\
\mathbf{0} & \lambda_{k} & \mathbf{1} & \ddots & \vdots \\
\vdots & \ddots & \ddots & \ddots & \mathbf{0} \\
\vdots & & \ddots & \lambda_{k} & \mathbf{1} \\
\mathbf{0} & \ldots & \ldots & \mathbf{0} & \lambda_{k}
\end{array}\right] \in C^{\boldsymbol{m}_{k} \times \boldsymbol{m}_{k}}
$$

The symbols $\boldsymbol{O}$, o and $\sim$, due to Bachmann and Landau (1927), which are also used by e.g. F. W. J. Olver [11] and A. Erd'elyi [12]. Concerning the definition and elementary properties of asymptotic series we refer to W. Wa-sow [9] and A. Erd'elyi [12].

Lemma2.1 (matrix function via Jordan canonical form). Let $\mathrm{f}$ be defined on $\sigma(\mathrm{P}), \mathrm{P} \in \mathrm{C}^{\mathrm{r} \times \mathrm{r}}$ and let $\mathrm{p}$ have the Jordan canonical form (2.5) subject to (2.6).Then

$$
\begin{gathered}
\mathbf{f}(\mathbf{P})=\mathbf{T} \mathbf{f}(\mathbf{J}) \mathbf{T}^{-1}=\mathbf{T} \operatorname{diag}\left(\mathbf{f}\left(\mathrm{J}_{1}\right), \mathbf{f}\left(\mathrm{J}_{2}\right), \cdots\right. \\
\left.\cdot, \mathbf{f}\left(\mathbf{J}_{\mathrm{s}}\right)\right) \mathbf{T}^{-1}
\end{gathered}
$$

Where

$$
\boldsymbol{f}\left(\boldsymbol{J}_{k}\right)=\left[\begin{array}{cccc}
f\left(\lambda_{k}\right) & \boldsymbol{f}^{(1)}\left(\lambda_{k}\right) & \ldots & \frac{f^{\left(m_{k}-1\right)}\left(\lambda_{k}\right)}{\left(m_{k}-1\right) !} \\
0 & f\left(\lambda_{k}\right) & \ddots & \vdots \\
\vdots & \ddots & \ddots & f^{(1)}\left(\lambda_{k}\right) \\
0 & \cdots & 0 & f\left(\lambda_{k}\right)
\end{array}\right] \in C^{m_{k} \times m_{k}}
$$

Proof: The proof of this lemma is already proved in [8]

A hypergeometric function is the sum of a hypergeometric series, which is defined as follows see e.g. F. W. J. Olver [11]. The hypergeometric function $\boldsymbol{p} \boldsymbol{F} \boldsymbol{q}\left(\mathrm{a} 1, \mathrm{a} 2, \cdots \mathrm{ap} ; \mathrm{b}_{1}, \mathrm{~b}_{2}, \cdots \mathrm{b}_{\mathrm{q}}\right.$; z) is defined by means of a hypergeometric series as

$$
\begin{aligned}
\boldsymbol{p F q}\left(\boldsymbol{a}_{1}, \boldsymbol{a}_{2}, \cdots \boldsymbol{a}_{\boldsymbol{p}} ; \boldsymbol{b}_{1}, \boldsymbol{b}_{2}, \cdots \boldsymbol{b}_{\mathrm{q}} ; \boldsymbol{z}\right) \\
=\sum_{n=0}^{\infty} \frac{\left(a_{1}\right) n\left(a_{2}\right) n \cdots\left(a_{P}\right) n}{\left(b_{1}\right) n\left(b_{2}\right) n \cdots\left(b_{q}\right) n} \frac{z^{n}}{n !} .
\end{aligned}
$$

Recall that the shifted factorial (a) $n$ is defined $b$

$N$ and $(a)_{0}=1$.

$$
(a)_{n}=a(a+1)(a+2) \cdots(a+n-1), n \in
$$

Gauss's hypergeometric equation is a second order differential equation with three regular singular points $\{0,1, \infty\}$, that is

$$
z(1-z) f^{\prime \prime}+[c-(1+a+b) z] f^{\prime}-a b f=0 .
$$

and has a solutions

$$
{ }_{2} \mathrm{~F}_{1}(\boldsymbol{a}, \boldsymbol{b} ; \boldsymbol{c} ; \boldsymbol{z})=\sum_{n=0}^{\infty} \frac{(\boldsymbol{a})_{n}(\boldsymbol{b})_{n}}{(\boldsymbol{c})_{n}} \frac{z^{n}}{n !}
$$

Theorem 2.2 for $\operatorname{Re} \boldsymbol{c}>\operatorname{Re} \boldsymbol{b}>\mathbf{0}$ we have

$$
\begin{aligned}
& { }_{2} \mathrm{~F}_{1}(a, b ; c ; z)= \\
& \quad \frac{\Gamma(c)}{\Gamma(b) \Gamma(c-b)} \int_{0}^{1} t^{b-1}(1-t)^{c-b-1}(1-z t)^{-a} d t,
\end{aligned}
$$

for all $\boldsymbol{z} \in \boldsymbol{C}$ and $|\boldsymbol{z}|<\mathbf{1}$.

The hypergeometric function with matrix $\operatorname{arguments} \mathrm{F}(\mathrm{A}, \mathrm{B} ; \mathrm{C} ; \mathrm{z})$, see L. J'odar and J. G. Sastre [6], is a solution of the differential equation

$$
\begin{gathered}
z(1-z) W^{(2)}-z A W^{(1)}+W^{(1)}(C+z(n-1) I)+n A W \\
=0
\end{gathered}
$$

which is defined by

$F(A, B ; C ; z)=$

$$
\begin{gathered}
\left(\int_{0}^{1}(1-t z)^{-A} t^{B-I}(1-t)^{C-B-I} d t\right) \Gamma^{-1}(B) \Gamma^{-1} \\
(C-B) \Gamma(C)
\end{gathered}
$$

Proposition 2.3 Let $\mathrm{f}(\mathrm{t})$ be a complex valued function of a real variable t such that $(i) f(t)$ is continuous on $(0, \infty)$,

$$
\text { (ii) } f(t) \sim \sum_{n=0}^{\infty} a^{\xi n-1} \text { as } t \rightarrow 0 \text { with } 0<\xi_{0}<\xi_{1}<\xi_{2}
$$

without loss of generality assume $a_{0} \neq 0$,

(iii) for some fixed $c>0 f(t)$

Then we have

$$
=O\left\{e^{c t}\right\} \text { as } t \rightarrow \infty \text {. }
$$

$$
\frac{d^{v}}{d \lambda_{k}^{v}}\left(\int_{0}^{\infty} e^{-\lambda_{k} t} f(t) d t\right) \sim \sum_{n=0}^{\infty} \frac{d_{n} \Gamma\left(\xi_{n}+v\right)}{\lambda_{k}^{\xi n+v}} \text { as } \operatorname{Re} \lambda_{k} \rightarrow
$$

$\infty \quad(2.11)$

where $d_{n}=(-1)^{v} a_{n}$ and

$$
\begin{aligned}
&\left|\arg \left(\lambda_{k}\right)\right| \leq \frac{\pi}{2}- \delta<\frac{\pi}{2} \text { for some } \delta \text { such that } 0<\delta \\
&<\frac{\pi}{2} .
\end{aligned}
$$

Proof: The proof of this proposition is already proved in [13]

Lemma 2.4 Suppose $Q$ is a positive stable matrix in $\mathrm{C}^{\mathrm{r} \times \mathrm{r}}$ and suppose also that $f(t) I \in \mathrm{C}^{\mathrm{r} \times \mathrm{r}}$, where $\mathrm{f}(\mathrm{t})$ is a function of a real variable $\mathrm{t}$ such that

(i) $f(t)$ is continuous on $(0, \infty)$.

(ii) $f(t) \sim \sum_{n=0}^{\infty} a_{n} t^{\xi n-1}$ as $t \rightarrow 0$

$$
0<\xi_{0}<\xi_{1}<\xi_{2}<\cdots \ldots
$$

(iii) for some fixed $c>0, f(t)=O\left\{e^{c t}\right\}$ as

$$
t \rightarrow \infty
$$

(iv) $Q=P$ has the Jordan canonical form subject to (2.5) and (2.6) with

$\left|\arg \left(\lambda_{k}\right)\right| \leq \frac{\pi}{2}-\delta<\frac{\pi}{2}$ for some $\delta$ such that

$0<$

$\delta<\frac{\pi}{2}$.

Then we have

$\boldsymbol{T}^{-1}\left[\int_{0}^{\infty} \boldsymbol{e}^{-\boldsymbol{Q} t} \boldsymbol{f}(\boldsymbol{t}) \mathrm{dt}\right] \mathrm{T} \sim \boldsymbol{\Phi}_{\boldsymbol{Q}}$ as $\operatorname{Re} \lambda_{\mathrm{k}} \rightarrow \infty$

Where $\boldsymbol{\Phi}_{\boldsymbol{Q}}=\operatorname{diag}\left(\boldsymbol{\Phi}_{1}, \boldsymbol{\Phi}_{2}, \cdots, \boldsymbol{\Phi}_{\boldsymbol{S}}\right)$ is a square block diagonal matrix in $\mathrm{C}^{\mathrm{r} \times \mathrm{r}}$ with blocks

$\Phi_{\mathrm{k}} \in \mathbb{C}^{m_{k} \times m_{k}}$.

$$
\Phi_{k}
$$$$
\left[\begin{array}{ccccc}
\sum_{n=0}^{\infty} \frac{d_{n} \Gamma\left(\xi_{n}\right)}{\lambda_{k}^{\xi n}} & \sum_{n=0}^{\infty} \frac{d_{n} \Gamma\left(\xi_{n}+1\right)}{\lambda_{k}^{\xi n+1}} & \ldots & \sum_{n=0}^{\infty} \frac{d_{n} \Gamma\left(\xi_{n}+m_{k}-1\right)}{\lambda_{k}^{\xi n+m_{k}-1}\left(m_{k}-1\right) !} \\
\mathbf{0} & \sum_{n=0}^{\infty} \frac{d_{n} \Gamma\left(\xi_{n}\right)}{\lambda_{k}^{\xi n}} & \ddots & \sum_{n=0}^{\infty} \frac{d_{n} \Gamma\left(\xi_{n}+1\right)}{\lambda_{k}^{\xi n+1}} \\
\vdots & \ddots & \ddots & \sum_{n=0}^{\infty} \frac{d_{n} \Gamma\left(\xi_{n}\right)}{\lambda_{k}^{\xi n}}
\end{array}\right]
$$

(2.14)

or we can write $\boldsymbol{\Phi}_{\boldsymbol{k}} \boldsymbol{a s}$ 


$$
\Phi_{k}=D_{k}+E_{k}=D_{k}\left[I_{m k}+D_{k}^{-1} E_{k}\right]
$$

where

$$
\left[\begin{array}{cclc}
\begin{array}{c}
D_{k}= \\
\sum_{n=0}^{\infty} \frac{\boldsymbol{d}_{n} \Gamma\left(\xi_{n}\right)}{\lambda_{k}^{\xi n}}
\end{array} & \mathbf{0} & \cdots & \mathbf{0} \\
\mathbf{0} & \sum_{\boldsymbol{n}=\mathbf{0}}^{\infty} \frac{\boldsymbol{d}_{n} \Gamma\left(\xi_{n}\right)}{\lambda_{k}^{\xi n}} & \ddots & \vdots \\
\vdots & \ddots & \ddots \sum_{n=0}^{\infty} \frac{\boldsymbol{d}_{n} \Gamma\left(\xi_{n}\right)}{\lambda_{k}^{\xi n}}
\end{array}\right] \in \mathbb{C}^{\boldsymbol{m}_{k} \times \boldsymbol{m}_{k}}
$$

And

$$
\left[\begin{array}{ccccc}
\mathbf{0} & \boldsymbol{E}_{\boldsymbol{k}}= & & \\
\mathbf{0} & \sum_{n=0}^{\infty} \frac{\boldsymbol{d}_{n} \Gamma\left(\xi_{n}+1\right)}{\lambda_{k}^{\xi n+1}} & \ldots & \sum_{n=0}^{\infty} \frac{\boldsymbol{d}_{n} \Gamma\left(\xi_{n}+r_{k}-1\right)}{\lambda_{k}^{\xi n+r_{k}-1}\left(r_{k}-1\right) !} \\
\vdots & \ddots & \ddots & \vdots \\
\vdots & & \ddots & \sum_{n=0}^{\infty} \frac{d_{n} \Gamma\left(\xi_{n}+1\right)}{\lambda_{k}^{\xi n+1}} \\
\mathbf{0} & \ldots & \ldots & 0
\end{array}\right] \in \mathbb{C}^{m_{k} \times m_{k}}
$$

Proof: The proof of this lemma is already proved in [13] .

3 On a matrix Hypergeometric Differential Equation.

In this section we apply the machinery of the previous

sections to show that in a certain sense

$$
F(a I, b I, C, z) \sim I \text { as } \operatorname{Re} \lambda \rightarrow \infty
$$$$
\text { and } F(a I, b I, \theta Q, z) \sim I \text { as } \theta \rightarrow \infty \text {, }
$$

with $Q$ a fixed matrix.

Theorem 3.1 Suppose $\mathrm{C}$ - bI $\in \mathbb{C}^{\mathrm{r} \times \mathrm{r}}$ has a Jordan canonical form subject to (2.5) and (2.6) then for any fixed matrices aI, bI in $\mathbb{C}^{\mathrm{r} \times \mathrm{r}}, \mathrm{b}>$ 0 we have

$$
T^{-1} F(a I, b I ; C ; z) T I \text { as } \operatorname{Re} \lambda_{k} \rightarrow \infty
$$

uniformly for $|\mathrm{z}| \leq \delta<1$ with $\delta$ a fixed number.

Proof Consider the following integral as a function of a matrix $\mathrm{C}$ $\varphi(\mathrm{C})=\int_{0}^{1}(1-z t)^{-a I} \mathrm{t}^{(\mathrm{b}-1) \mathrm{I}}(1-\mathrm{t}) \mathrm{C}^{-(\mathrm{b}+1) \mathrm{I}} d t$.

$$
\text { (3.2) }
$$

Setting $\quad 1-\mathrm{t}=\mathrm{e}^{-\mathrm{u}}, \mathrm{dt}=\mathrm{e}^{-\mathrm{u}} \mathrm{du} \quad$ when $\mathrm{t}=1 \Rightarrow \mathrm{u} \rightarrow \infty$, also when $\mathrm{t}=0 \Rightarrow \mathrm{u}=0$

so we have

$$
\begin{aligned}
& \left.\varphi(C)=\int_{0}^{\infty}\left(1+\mathrm{z}\left(\mathrm{e}^{-\mathrm{u}}-1\right)\right)^{-\mathrm{aI}}\left(1-\mathrm{e}^{-\mathrm{u}}\right)^{(\mathrm{b}-1) \mathrm{I}} \mathrm{e}^{-\mathrm{u}(\mathrm{C}-} \quad(\mathrm{b}+1) \mathrm{I}\right) \mathrm{e}^{-\mathrm{u}} \mathrm{dt} . \\
& =\int_{0}^{\infty}\left(1+\mathrm{z}\left(\mathrm{e}^{-\mathrm{u}}-1\right)\right)^{-\mathrm{aI}}\left(1-\mathrm{e}^{-\mathrm{u}}\right)^{(\mathrm{b}-1) \mathrm{I}} \mathrm{e}^{-\mathrm{u}(\mathrm{C}-\mathrm{bl})} \mathrm{dt} \text {. }
\end{aligned}
$$

Note that

$$
\left(1+z\left(e^{-u}-1\right)\right)^{-a I}=\left(1+z\left(\frac{-u}{1 !}+\frac{u^{2}}{2 !}-\frac{u^{3}}{3 !}+\frac{u^{4}}{4 !} \ldots\right)\right)^{-a I}
$$

Therefore

Also

$$
\left(1+z\left(e^{-u}-1\right)\right)^{-a I} \sim I \text { as } \mathrm{u} \rightarrow 0
$$

$$
\left(1+e^{-u}\right)^{-(b-1) I}=\left(1-1+\frac{-u}{1 !}+\frac{u^{2}}{2 !}-\frac{u^{3}}{3 !}+\frac{u^{4}}{4 !} \ldots\right)^{(b-1) I}
$$$$
u^{(b-1)}\left(1-\frac{-u}{1 !}+\frac{u^{2}}{2 !}-\frac{u^{3}}{3 !}+\frac{u^{4}}{4 !} \ldots \ldots\right)^{(b-1)} I
$$

When $\mathrm{u} \longrightarrow 0$ we have

$$
\left(1+e^{-u}\right)^{(b-1) I} \sim \mathrm{u}^{\mathrm{b}-1} I
$$

thus

$$
\left(1+z\left(e^{-u}-1\right)\right)^{-a I}\left(1-e^{-u}\right)^{(\mathrm{b}-1) \mathrm{I}} \sim \mathrm{u}^{\mathrm{b}-1} I d t
$$

$$
\text { as } \mathrm{u} \longrightarrow 0 \text {. }
$$

Now lemma 2.4 implies that

$$
\begin{aligned}
\mathrm{T}^{-1} & {\left[\int_{0}^{1}(1-z t)^{-a I} \mathrm{t}^{(\mathrm{b}-1)} \mathrm{I}(1-\mathrm{t})^{\mathrm{C}-(\mathrm{b}-1) \mathrm{I}}\right] \mathrm{T} } \\
& =\int_{0}^{1}(1-z t)^{-u I_{k}} t^{(b-1) I_{k}}(1-t)^{J_{k}-I} d t \sim \quad \Phi_{\mathrm{C}-\mathrm{bI}} \quad \text { as }
\end{aligned}
$$

$\operatorname{Re} \lambda_{\mathrm{k}} \rightarrow \infty$

Note that by letting $\mathrm{a}=0$ in equation (3.3), we obtain the following representation for the Beta matrix. Namely

$$
\begin{aligned}
\beta(\mathrm{C}-b I, b I) & =\int_{0}^{1} t^{(b-1) I}(1-t)^{C-(b+1) I} d t \\
& =\Gamma^{-1}(\mathrm{C}) \Gamma(\mathrm{C}-b I) \Gamma(b I)
\end{aligned}
$$

and obtain

$\mathrm{T}^{-1}[\beta(\mathrm{Bi}, \mathrm{C}-\mathrm{bI})] \mathrm{T}=\mathrm{T}^{-1}\left[\int_{0}^{1} \mathrm{t}^{(\mathrm{b}-1) \mathrm{I}}(1-\mathrm{t})^{\mathrm{C}-(\mathrm{b}+1) \mathrm{I}} \mathrm{dt}\right] \mathrm{T}$

$$
\begin{aligned}
& =\int_{0}^{1} \mathrm{t}^{(\mathrm{b}-1) \mathrm{I}}(1-\mathrm{t})^{\mathrm{J}_{\mathrm{k}}-\mathrm{I}} \mathrm{dt} \\
& \quad \sim \Phi_{\mathrm{C}-\mathrm{bI}} \text { as } \operatorname{Re} \lambda_{\mathrm{k}} \rightarrow \infty \mathrm{k}=1,2 \ldots \ldots
\end{aligned}
$$

It is readily observed that

$\mathrm{F}(a I, b I ; C ; z)$

$=\left(\int_{0}^{1}(1-t z)^{-a I} \mathrm{t}^{(\mathrm{b}-1) I}(1-\mathrm{t})^{\mathrm{C}-(\mathrm{b}+1) \mathrm{I}} d t\right) \Gamma^{-1}(\mathrm{bI}) \Gamma^{-1}(\mathrm{C}-b I) \Gamma(\mathrm{C})$ (3.5)

in the sense that $\mathrm{T}^{-1} \mathrm{~F}(a I, b I ; C ; z) \mathrm{T} \sim \mathrm{I}$.

Example Let $\mathrm{C}=\mathrm{c} \in(0, \infty)$ in the equation (3.5), then for any fixed a and

$$
\begin{gathered}
\mathrm{b}>0 \text { we have } \\
\varphi(\mathrm{C})=\int_{0}^{1} t^{b-1}(1-t)^{\mathrm{c}-\mathrm{b}-1}(1-z t)^{-\mathrm{a}} d t .
\end{gathered}
$$

Setting $1-\mathrm{t}=\mathrm{e}^{-\mathrm{u}}, \mathrm{dt}=\mathrm{e}^{-\mathrm{u}} d u$ when

$\mathrm{t}=1 \Rightarrow \mathrm{u} \rightarrow \infty$, also when $\mathrm{t}=0 \Rightarrow \mathrm{u}=0$

so we have

$\varphi(\mathrm{C})=\int_{0}^{1}\left(1+z\left(e^{-u}-1\right)\right)^{-a}\left(1-1-e^{-u}\right)^{\mathrm{b}-1} \mathrm{e}^{-u(c-b)} d u$.

Note that

$\left(1+z\left(e^{-u}-1\right)\right)^{-a}\left(1-1-e^{-u}\right)^{\mathrm{b}-1} \sim u^{b-1}$

as $\mathrm{u} \rightarrow 0$

Now Watson's lemma implies that

$\int_{0}^{1}\left(1+z\left(e^{-u}-1\right)\right)^{-a}\left(1-1-e^{-u}\right)^{\mathrm{b}-1} \mathrm{e}^{-u(c-b)} d u \sim \frac{\Gamma(b)}{(b-c)^{b}}$ as $\mathrm{c} \rightarrow \infty$

or

$$
\int_{0}^{1} \mathrm{t}^{\mathrm{b}-1}(1-\mathrm{t})^{\mathrm{c}-\mathrm{b}-1}(1-\mathrm{zt})^{-\mathrm{a}} \mathrm{dt} . \sim \frac{\Gamma(\mathrm{b})}{(\mathrm{b}-\mathrm{c})^{\mathrm{b}}}
$$

$$
\text { as } c \rightarrow \infty
$$

By assuming $\mathrm{a}=0$ in (3.6) we obtain

$$
\beta(b, c-b)=\int_{0}^{1} t^{b-1}(1-t)^{c-b-1} d t \sim \frac{\Gamma(b)}{(b-c)^{b}}
$$

By (3.6) and (3.7) we derive asymptotic expansion for ${ }_{2} \mathrm{~F}_{1}(\mathrm{a}, \mathrm{b} ; \mathrm{c} ; \mathrm{z})$ when $\mathrm{c}$

Approaches infinity,

$$
{ }_{2} \mathrm{~F}_{1}(\mathrm{a}, \mathrm{b} ; \mathrm{c} ; \mathrm{z}) \sim 1 \text { as } \mathrm{c} \rightarrow \infty
$$

Corollary 3.2 Suppose $C-b I=\theta Q$ where $\theta \in(0, \infty)$ and $Q$ is a constant matrix and has a Jordan canonical form subject to (2.5) and (2.6) then

$\mathrm{T}^{-1}\left[\int_{0}^{1}(1-\mathrm{zt})^{-\mathrm{aI}} \mathrm{t}^{(\mathrm{b}-1) \mathrm{I}}(1-\mathrm{t})^{\mathrm{c}-(\mathrm{b}+1) \mathrm{I}} \mathrm{dt}\right] \mathrm{T} \sim \Psi_{\theta \mathrm{Q}}$ as $\theta \rightarrow$ $\infty \quad$ (3.9)

Where $\Psi_{\theta Q}=\operatorname{diag}\left(\Psi 1, \Psi 2, \cdots, \Psi_{S}\right)$ is a square block diagonal matrix in $\mathbb{C}^{\mathrm{r} \times \mathrm{r}}$, with blocks $\Psi_{k} \in \mathbb{C}^{m_{k} \times m_{k}}$,

$\Psi \mathrm{k}=$

$$
\left[\begin{array}{cccc}
\sum_{n=0}^{\infty} \frac{a_{n} \Gamma\left(\xi_{n}\right)}{\theta^{\xi n}} & \sum_{n=0}^{\infty} \frac{d_{n} \lambda_{k} \Gamma\left(\xi_{n}+1\right)}{\left(\theta \lambda_{k}\right)^{\xi_{n}+1}} & \ldots & \sum_{n=0}^{\infty} \frac{d_{n} \lambda_{k} \Gamma\left(\xi_{n}+m_{k}-1\right)}{\left(\theta \lambda_{k}\right)^{\xi n+m_{k}-1}\left(m_{k}-1\right) !} \\
0 & \sum_{n=0}^{\infty} \frac{a_{n} \Gamma\left(\xi_{n}\right)}{\theta^{\xi n}} & \ddots & \vdots \\
\vdots & \ddots & \ddots & \sum_{n=0}^{\infty} \frac{d_{n} \lambda_{k} \Gamma\left(\xi_{n}+1\right)}{\left(\theta \lambda_{k} \xi_{n}+1\right.} \\
0 & \ldots & 0 & \sum_{n=0}^{\infty} \frac{a_{n} \Gamma\left(\xi_{n}\right)}{\theta^{\xi n}}
\end{array}\right]
$$

Moreover,

$$
\mathrm{T}^{-1} \mathrm{~F}(\mathrm{aI}, \mathrm{bI} ; \theta \mathrm{C} ; \mathrm{z}) \mathrm{T} \sim \mathrm{I} \text { as } \theta \rightarrow \infty
$$

Uniformly for $|\mathrm{z}| \leq \delta<1$ with $\delta$ a fixed number.

Proof: By Watson's lemma we have

$$
\underset{\theta \rightarrow \infty}{\int_{0}^{1}(1-z t)^{-a} t^{(b-1)}(1-t)^{\left(\theta \lambda_{k}\right)-1} d t \sim \sum_{n=0}^{\infty} \frac{a_{n} \Gamma\left(\xi_{n}\right)}{\left(\theta \lambda_{k}\right)^{\xi}}}
$$

and by proposition 2.3 as $\theta \rightarrow \infty$ we get

$$
\begin{aligned}
& \frac{\mathrm{d}^{\mathrm{v}}}{\mathrm{d}^{\mathrm{v}}}\left(\int_{0}^{1} 1-\mathrm{zt}\right)^{-\mathrm{at}} \quad \mathrm{t}^{(\mathrm{b}-1)} \quad(1-\mathrm{t})^{\left(\theta \lambda_{\mathrm{k}}\right)-1} \mathrm{dt} \quad{ }^{2} \quad \text { } \\
& \sum_{\mathrm{n}=0}^{\infty} \frac{\mathrm{d}_{\mathrm{n}} \lambda_{\mathrm{k}}^{\mathrm{v}} \Gamma\left(\xi_{\mathrm{n}}+\mathrm{v}\right)}{\left(\theta \lambda_{\mathrm{k}}\right)_{\mathrm{n}+\mathrm{v}}} \text {. }
\end{aligned}
$$


$\int_{0}^{1}(1-\mathrm{zt})^{-\mathrm{aI}_{\mathrm{k}}} \mathrm{t}^{(\mathrm{b}-1) \mathrm{I}_{\mathrm{k}}}(1-\mathrm{t})^{\theta \mathrm{J}_{\mathrm{k}}-1} \mathrm{dt} \sim \Psi_{\mathrm{k}} \quad$ as $\quad \theta \rightarrow \infty$, and the results (3.9) and (3.10) follow.

\section{Conclusion}

We show that

and

$$
F(a I, b I, C, z) \sim I \text { as } \operatorname{Re} \lambda \rightarrow \infty
$$

with $Q$ a fixed matrix

\section{References:}

[1]- J'odar L. and Cort'e J. G. , Some Properties Of Gamma And Beta Matrix Function, Appl . Math. Lett, 11, (1998), 89-93

[2]- J'odar L . and Company R. Hermite Matrix Polynomials And Second Order Matrix Differential Equation, Approximation Theory and its Application 12(2), 20-30, (1996).

[3]- Defez E. and J'odar, L. Some Applications of the Hermite Matrix Polynomials Series Expansions, Journal Of Computational Applied Mathematics 99, 105-117, (1998).

[4]- Jodar L. and Sastre J. The growth Of Laguerre Matrix Polynomials On Bounded Intervals, appl. math. lett, 13, (2000), 21-26.

[5]- Defez E. , and J'odar L . Chebyshev Matrix Polynomials And Second Order Matrix Differential Equation. Utilitas Mathematica , (2002). 61. 107-123.

[6]- J'odar L. and Cort'es J. G. On the Hypergeometric Matrix Function, J. Comp. Appl . Math.99, (1998),205-217.

[7]- Batahan R. S. Generalized Form Of Hermite Matrix Polynomials Via the Hypergeometric Matrix Function, Advances In Linear Algebra And Matrix Theory, (2014) ,4,134-141. 